\section{Der Durchblick}

\section{BACHELOR-STUDIENGÄNGE Seit 2001 können}

Physiotherapeuten in Deutschland ihren Bachelor machen. So vielfältig der Beruf, so vielfältig die Studienangebote. Um den Überblick zu bewahren, haben wir für Sie die wichtigsten Infos gesammelt.

n fast allen europäischen Ländern ist die Ausbildung zum Physiotherapeuten ein Hochschulstudium. Seit 2001 können examinierte Physiotherapeuten auch in Deutschland einen Bachelorabschluss erwerben. Mittlerweile gibt es sogar Masterabschlüsse in der Physiotherapie, die eine Promotion erleichtern. Die Angebotspalette ist sehr groß: Man kann im Anschluss an die Berufsfachschule (=ausbildungsaufbauend) in Vollzeit oder berufsbegleitend in Teilzeit studieren oder während der Ausbildung parallel studieren (= ausbildungsintegrierend). Seit dem Wintersemester 2010/2011 können sich Abiturienten erstmals für ein grundständiges, also primärqualifizierendes Studium mit deutschem Abschluss immatrikulieren. Bei ihrer Entscheidung sollten die angehenden Studenten auch den jeweiligen Abschluss beachten. Denn es gibt neben dem deutschen auch einen niederländischen Bachelor. Diesen müssen sie für ein deutsches Masterstudium erst anerkennen lassen. Vergleichen und informieren lohnt sich, wenn es um die Wahl des Studiums geht.

Kathrin Bauer

\section{$\Rightarrow$ Im Internet finden Sie unter www.thieme-connect.de/ejournals/toc|} physiopraxis > „Ausgabe 4/2011“ eine Adressliste mit allen Hochsschulen inklusive der Kooperationsschulen. In der nächsten physiopraxis geben wir Ihnen einen Überblick über die Master-Studiengänge.

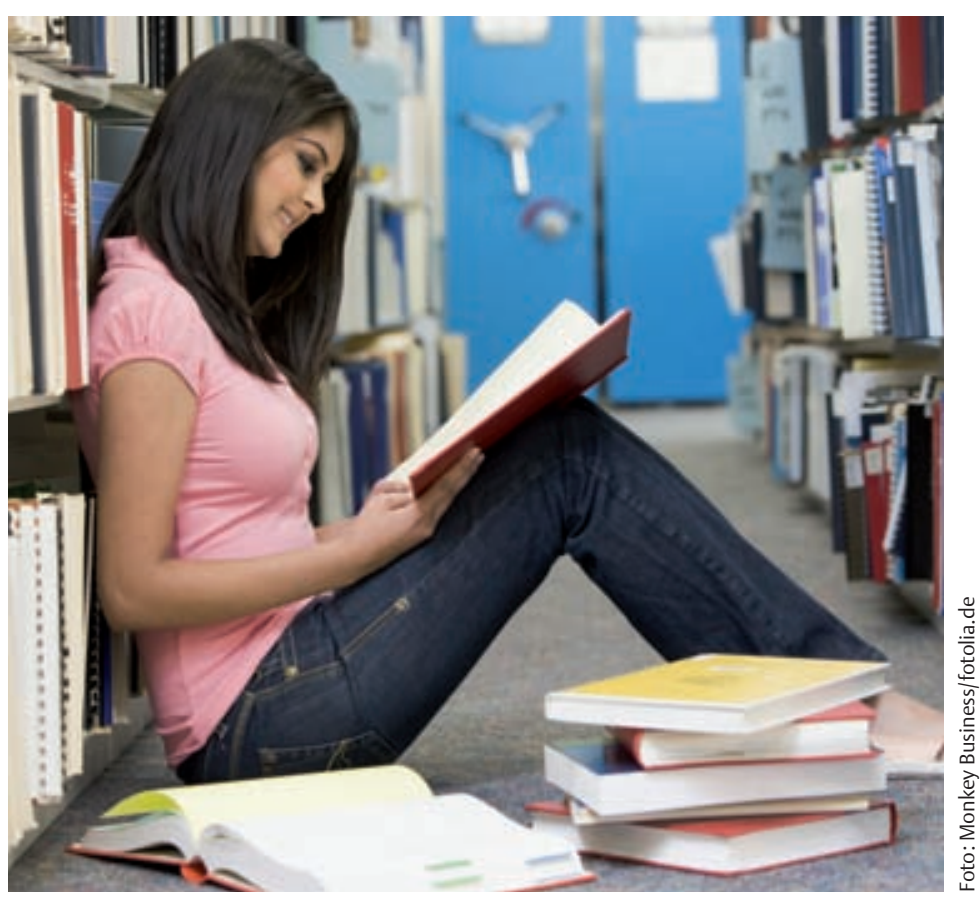

\section{DEUTSCHLAND}

primärqualifizierend

1 Hochschule Fresenius www.hs-fresenius.de Studienstandort: Hamburg, Köln oder Idstein Studiengangsleiterin: Hester van Wijnen

2 Alice Salomon Hochschule Berlin www.ash-berlin.eu, Studiengangsleiterin: Prof. Dr. Jutta Räbiger

3 Hochschule für Gesundheit Bochum www.fh-gesundheit.de

Studiengangsleiter: Prof. Dr. Christian Grüneberg

4 Hochschule Fulda

www.hs-fulda.de

Philipps-Universität Marburg

www.uni-marburg.de

Studienstandort: Fulda und Marburg

Studiengangsleiter: Prof. Dr. Klaus Stegmüller, Udo Wolf

$5 \quad$ Hochschule für angewandte Wissenschaften Bamberg (früher: FH Schloss Hohenfels), www.fh-schloss-hohenfels.de Studiengangsleiter: Prof. Dr. Konstantin Karanikas

6 SRH Hochschule Heidelberg www.fh-heidelberg.de

Studiengangsleiterin: Prof. Dr. Mieke Wasner

7 VPT Hochschule für Gesundheitswissenschaften Fellbach (in Gründung), www.vpt-hochschule.de Studiengangsleiter: N.N.

\section{ausbildungsintegriert}

8 Fachhochschule Kiel

Fachbereich Soziale Arbeit und Gesundheit www.fh-kiel.de, Studiengangsleiterin: Prof. Dr. Heidi Höppner

9 DIPLOMA Private Hochschule www.diploma.de

Studienstandort: Schwentinental, Berlin, Friedrichshagen, Hannover, Leipzig, Kassel, Bad Sooden-Allendorf, Bonn, Plauen, Mannheim, Aalen, Baden-Baden oder München Studiengangsleiter: unterschiedlich je nach Studienstandort

10 MSH Medical School Hamburg www.medicalschool-hamburg.de Studiengangsleiter: Prof. Dr. Thomas Gehrke

11 Hochschule 21 Buxtehude www.hs21.de Studiengangsleiterin: Prof. Dr. Barbara Zimmermann

12 Hochschule Emden/Leer Fachbereich Soziale Arbeit und Gesundheit www.hs-emden-leer.de Studiengangsleiterin: Prof. Dr. Ruth Haas

13 Fachhochschule Osnabrück www.fh-osnabrueck.de, Studiengangsleiterin: Prof. Dr. Hilke Hansen, Professoren der Physiotherapie: Prof. Dr. Harry von Piekartz, Prof. Dr. Christoff Zalpour

14 HAWK Hildesheim/Holzminden/Göttingen www.hawk-hhg.de Studiengangsleiter: Prof. Dr. Axel Schäfer Studiendekan: Prof. Dr. Bernhard Borgetto

15 Hochschule Lausitz Senftenberg www.hs-lausitz.de Studiendekan: Prof. Dr. Sven Michel

16 Dresden International University www.dresden-international-university.com Studiengangsleiter: Prof. Dr. Hans Wiesmeth 


seit ab a

nein

8 Semester

WS

Bachelor of Science primärqualifizierend

Bachelor of Science primärqualifizierend

Bachelor of Science primärqualifizierend

7 Semester ja

WS

Bachelor of Science primán

Bachelor of Science primärqualifizierend

7 Semester nein

WS

(1)

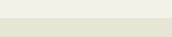

$\begin{array}{ll}\text { WS 10/11 } & \text { Idstein: 445,- } € \text { pro Monat } \\ \text { WS 10/11 } & \text { Hamburg: 490,- } € \text { pro Monat } \\ \text { WS 11/12 } & \text { Köln: 490,- } € \text { pro Monat }\end{array}$

240

WS 11/12 Köln: 490,- € pro Monat Köln: 25

Hamburg: 50

WS 11/12 keine Studiengebühren

ca. 40

7 Semester nein

WS $10 / 11$

$300,-€$ pro Semester

ab WS11/12 keine Studien-

50

210

gebühren mehr

WS

\begin{tabular}{|c|c|c|c|c|c|c|c|}
\hline Bachelor of Science & primärqualifizierend & $\begin{array}{l}7 \text { Semester } \\
\text { WS }\end{array}$ & nein & WS $10 / 11$ & 495,- $€$ pro Monat & 30 & 210 \\
\hline Bachelor of Science & primärqualifizierend & $\begin{array}{l}7 \text { Semester } \\
\text { WS }\end{array}$ & nein & WS $10 / 11$ & 530,- $€$ pro Monat & 35 & 210 \\
\hline Bachelor of Science & primärqualifizierend & $\begin{array}{l}7 \text { Semester } \\
\text { SS und WS }\end{array}$ & nein & SS 11 & $480,-€$ pro Monat & 35 & 210 \\
\hline
\end{tabular}

\begin{tabular}{|c|c|c|c|c|c|c|c|}
\hline Bachelor of Science & ausbildungsintegriert & $\begin{array}{l}6 \text { Semester in } \\
4,5 \text { Jahren } \\
\text { WS }\end{array}$ & ja & WS $01 / 02$ & keine Studiengebühren & 60 & 180 \\
\hline Bachelor of Arts & $\begin{array}{l}\text { ausbildungsintegriert } \\
\text { bzw. berufsbe- } \\
\text { gleitend für exam. PT } \\
\text { (als Fernstudium) }\end{array}$ & $\begin{array}{l}4 \text { Semester bzw. } \\
5 \text { Semester } \\
\text { SS und WS }\end{array}$ & $\begin{array}{l}\text { ausbildungs- } \\
\text { integriert: ja, } \\
\text { berufsbe- } \\
\text { gleitend: nein }\end{array}$ & WS $08 / 09$ & 197,- € pro Monat & unbegrenzt & 180 \\
\hline
\end{tabular}

\begin{tabular}{|c|c|c|c|c|c|c|c|}
\hline Bachelor of Science & ausbildungsintegriert & $\begin{array}{l}7 \text { Semester in } \\
4,5 \text { Jahren } \\
\text { WS }\end{array}$ & ja & WS $10 / 11$ & $\begin{array}{l}4 \text { Semester: } 200,-€ \text { pro Monat } \\
3 \text { Semester: } 450,-€ \text { pro Monat }\end{array}$ & 25 & 180 \\
\hline Bachelor of Science & ausbildungsintegriert & $\begin{array}{l}8 \text { Semester } \\
\text { WS }\end{array}$ & ja & WS 06/07 & 350,- $€$ pro Monat & $\begin{array}{l}\text { pro Koopera- } \\
\text { tionsschule } 25\end{array}$ & 180 \\
\hline Bachelor of Science & $\begin{array}{l}\text { ausbildungsintegriert } \\
\text { bzw. ausbildungsauf- } \\
\text { bauend in Vollzeit für } \\
\text { exam. PT }\end{array}$ & $\begin{array}{l}4,5 \text { Jahren bzw. } \\
2 \text { Jahre } \\
\text { WS }\end{array}$ & ja & WS 09/10 & $\begin{array}{l}\text { während der Ausbildung 83,- } € \\
\text { pro Semester, im Vollzeitstu- } \\
\text { dium 500,- € pro Semester }\end{array}$ & 40 & 180 \\
\hline Bachelor of Science & ausbildungsintegriert & $\begin{array}{l}\text { voraussichtlich: } 8 \\
\text { Semester } \\
\text { voraussichtlich: SS }\end{array}$ & $\begin{array}{l}\text { ja } \\
\text { in Planung }\end{array}$ & SS 12 & $\begin{array}{l}\text { während der Ausbildung 50,- } € \\
\text { pro Semester, im Vollzeitstu- } \\
\text { dium 500,- } € \text { pro Semester }\end{array}$ & ca. 20 & $\begin{array}{l}\text { in Pla- } \\
\text { nung }\end{array}$ \\
\hline Bachelor of Science & $\begin{array}{l}\text { ausbildungsintegriert } \\
\text { bzw. ausbildungsauf- } \\
\text { bauend in Vollzeit für } \\
\text { exam. PT }\end{array}$ & $\begin{array}{l}4,5 \text { Jahren bzw. } \\
1,5 \text { Jahre } \\
\text { SS und WS }\end{array}$ & ja & SS 01 & $500,-€$ pro Semester & $\begin{array}{l}\text { im SS und WS } \\
\text { zusammen } \\
100\end{array}$ & 180 \\
\hline Bachelor of Science & $\begin{array}{l}\text { ausbildungsintegriert } \\
\text { ab WS11/12: } \\
\text { NC-Studiengang }\end{array}$ & $\begin{array}{l}9 \text { Semester in } \\
4,5 \text { Jahren } \\
\text { WS }\end{array}$ & $\begin{array}{l}\text { nein, freie } \\
\text { Wahl der BFS }\end{array}$ & WS 05/06 & 117,- € pro Semester & 50 & 180 \\
\hline Bachelor of Science & ausbildungsintegriert & $\begin{array}{l}8 \text { Semester in } \\
4 \text { Jahren } \\
\text { im April }\end{array}$ & ja & April 2011 & insgesamt 9.900,- $€$ & 20 & 180 \\
\hline
\end{tabular}


17 SRH Fachhochschule für Gesundheit Gera

www.gesundheitshochschule.de, Studiengangsleiter: Prof. Dr. Jan Mehrholz

18 Knowledge Foundation@Reutlingen University Reutlingen

Bachelor of Science ausbildungsintegriert www.kfru.de, Studiengangsleiter: Prof. Dr. Hans-Peter Baumeister

\section{ausbildungsaufbauend: Vollzeit}

19 Hochschule Emden/Leer

Fachbereich Soziale Arbeit und Gesundheit

www.hs-emden-leer.de

Studiengangsleiterin: Prof. Dr. Ruth Haas

20 Fachhochschule Osnabrück

www.fh-osnabrueck.de, Studiengangsleiterin: Prof. Dr. Hilke Hansen

Professoren der Physiotherapie: Prof. Dr. Harry von Piekartz, Prof. Dr. Christoff Zalpour

21 HAWK Hildesheim/Holzminden/Göttingen

www.hawk-hhg.de

Studiengangsleiter: Prof. Dr. Axel Schäfer

Studiendekan: Prof. Dr. Bernhard Borgetto

22 Hochschule Fulda

www.hs-fulda.de

Philipps-Universität Marburg

www.uni-marburg.de, Studienstandorte: Fulda und Marburg

Studiengangsleiter: Prof. Dr. Klaus Stegmüller, Udo Wolf

ausbildungsaufbauend: berufsbegleitend

23 DIPLOMA Private Hochschule

www.diploma.de, Studienstandort: Schwentinental, Berlin, Friedrichshagen, Hannover, Leipzig,

Bachelor of Arts

Kassel, Bad Sooden-Allendorf, Bonn, Plauen, Mannheim, Aalen, Baden-Baden oder München

Studiengangsleiter: Unterschiedlich je nach Studienstandort

24 MSH Medical School Hamburg

www.medicalschool-hamburg.de, Studiengangsleiter: Prof. Dr. Thomas Gehrke

25 Hochschule Fresenius

www.hs-fresenius.de

Studienstandort: Hamburg oder Idstein/Frankfurt

Studiengangsleiterin: Maria Geißler

26 HAWK Hildesheim/Holzminden/Göttingen

www.hawk-hhg.de, Studiengangsleiter: Prof. Dr. Axel Schäfer

Studiendekan: Prof. Dr. Bernhard Borgetto

27 Dresden International University www.dresden-international-university.com, Studienstandorte: Dresden und Stuttgart Wissenschaftliche Leitung: Prof. Dr. habil., Dr. h. c. mult. Klaus Steinbrück, Hans Hartogh, Dr. Kornelia Möser

28 SRH Fachhochschule für Gesundheit Gera www.gesundheitshochschule.de, Studiengangsleiter: Prof. Dr. Jan Mehrholz

29 Hochschule für angewandte Wissenschaften Bamberg, (früher: FH Schloss Hohenfels) www.fh-schloss-hohenfels.de, Studiengangsleiterin: Prof. Dr. Gabriele Hanne-Behnke

Kooperationspartner der „Internationale Hogeschool Fysiotherapie Thim van der Laan“

30 ecolea | Private Berufliche Schule

www.ecolea.de, Studienstandorte: Stralsund, Rostock-Warnemünde oder Schwerin und jeweils Nieuwegein (NL), Studiengangsleiter: Michael Erdmann

31 Döpfer-Akademie Schwandorf www.doepfer-schulen.de, Studienstandorte: Hamburg, Köln, Nürnberg, Regensburg oder München und jeweils Nieuwegein (NL), Studiengangsleiter: Michael Karp

32 IEB - Institut für Erwachsenenbildung Rheine www.das-ieb.de, Studienstandort: Rheine Studiengangsleiter: Jörg Stifter

33 Timmermeister Schule Münster www.timmermeister-schule.de Studienstandorte: Münster und und Nieuwegein (NL) Ausbildungsleiter: Peter Stracke

34 Heimerer Akademie GmbH www.heimerer.de, Studienstandort: Leipzig

Studiengangsleiterin: Susanne Bahn
Bachelor of

Physiotherapy (NL)

Bachelor of

Physiotherapy (NL)

Bachelor of

Physiotherapy (NL)

Bachelor of

Physiotherapy (NL)

Bachelor of

Physiotherapy (NL)
Bachelor of Science

Bachelor of Science

Bachelor of Science

(in

ausbildungsintegriert bzw. ausbildungsaufbauend in Vollzeit für exam. PT

ausbildungsaufbauend in Vollzeit

ausbildungsintegriert bzw. ausbildungsaufbauend in Vollzeit für exam. PT

ausbildungsaufbauend in Vollzeit

ausbildungsintegriert bzw. berufsbegleitend für exam. PT (als Fernstudium)

Bachelor of Science berufsbegleitend

Bachelor of Science berufsbegleitend

Bachelor of Science berufsbegleitend

Bachelor of Science berufsbegleitend

Bachelor of Science berufsbegleitend

Bachelor of Science berufsbegleitend

ausbildungsintegriert bzw. berufsbegleitend für exam. PT

ausbildungsintegriert bzw. berufsbegleitend für exam. PT

ausbildungsintegriert bzw. berufsbegleitend für exam. PT

ausbildungsintegriert bzw. berufsbegleitend für exam. PT

ausbildungsintegriert bzw. berufsbegleitend für exam. PT 


\section{Dauer und Beginn}

7 Semester in 4,5 Jahren

WS

8 Semester

im November

4,5 Jahre bzw. 2 Jahre

WS

ja

Kooperationsschulen

seit/ab

Kosten

WS 07/08 4 Semester: 125,- € pro Monat

3 Semester: 320,- $€$ pro Monat

nein, freie Wahl der BFS

ja

2009

6 Semester: $120,-€$ pro Monat

2 Semester: 395,- € pro Monat

WS 09/10 während der Ausbildung 83,- € pro

40

Semester, im Vollzeitstudium 500,- $€$

pro Semester

$\begin{array}{ll}3 \text { Semester } & \text { ja, es werden auch PTs von } \\ \text { SS und WS } & \text { anderen BFS aufgenommen }\end{array}$

4,5 Jahre bzw. 1,5 Jahre

SS und WS

ja

WS 01/02 500,- $€$ pro Semester

ca. 25

180

SS 01

während der Ausbildung 50,- $€$ pro $\quad$ im SS und WS

180

Semester, im Vollzeitstudium 500,- $€$ zusammen 100 pro Semester

nein

WS 01/02 keine Studiengebühren

30

180

80

180

180
6 Semester

WS ausbildungsintegriert: ja

berufsbegleitend: nein

5 Semester

SS und WS

9 Semester, verkürzt: 7 Semester

nein

WS

4 Semester

WS

\section{2,5 Jahre}

WS und SS

6 Semester in 3 Jahren

nächster Einstieg:

22. November 2011

6 Semester

WS

6 Semester

WS

\section{ja, es werden auch PTs von} anderen BFS aufgenommen

\section{WS 08/09 197,- $€$ pro Monat}

unbegrenzt

180

Points

45

4 Jahre bzw. 2,5 Jahre

ja

Mai 2009

insgesamt 4.500-5.500,- €

25

4 Jahre bzw. 30 Monate

ja

1999

insgesamt 5.000-5.500,- €

32

verschieden, je nach Studienstandort

4

ja

Oktober insgesamt 4.998-5.500,- $€$

32

240

2008

ja

April 2007 insgesamt 5.200-5.500,- €

30

240

SS

4 Jahre bzw. 2,5 Jahre im März, 2011 erstmalig im

September
24 


\section{physiospektrum}

\section{Alle Studienorte}
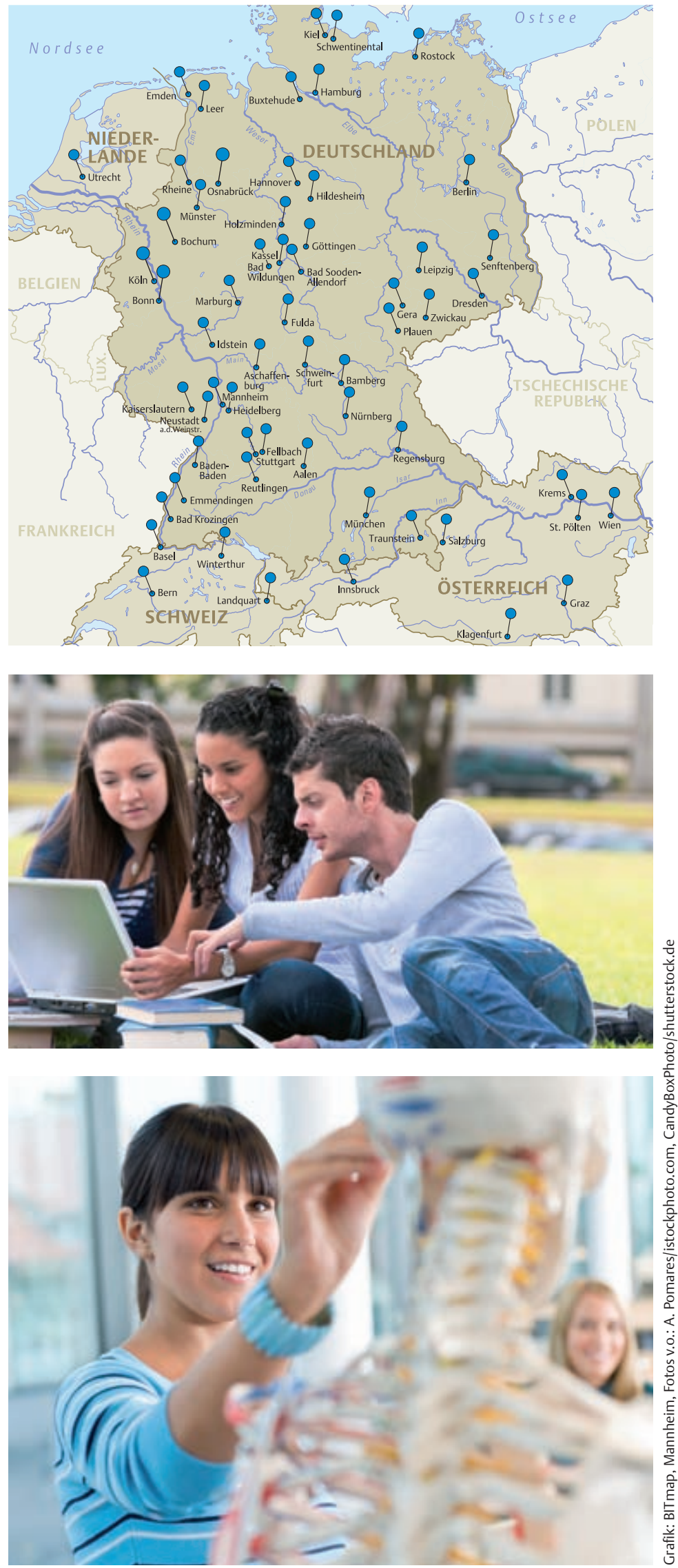

\section{Nr. Hochschule/Schule}

35 Bringmann Akademie

www.bringmann-akademie.de

Studienstandorte: Bad Wildungen und Nieuwegein (NL) Studiengangsleiter: Bertram Hahn

36 Hans-Weinberger-Akademie (HWA)

www.hwa-online.de

Studienstandorte: Schweinfurt, Aschaffenburg und Nieuwegein (NL)

Studiengangsleiterin: Dr. Gabriele Güthle

37 Akademie für Gesundheitsfachberufe Pfalz www.akademie-pfalz.de

Studienstandort: Kaiserslautern oder

Neustadt an der Weinstraße

Studiengangsleiter: Konstantin Beinert, Msc

38 Physiotherapieschule Emmendingen www.physio-em.de

Freiburger Lehrinstitute für Physikalische Therapie (LFK) Bad Krozingen, www.Ifk-physio.de

Studienstandorte: Emmendingen und Nieuwegein (NL) Studiengangsleiter: Gunther Speth

39 Chiemsee-Schule Zimmermann Traunstein www.chiemsee-schule-zimmermann.de Studienstandort: Traunstein Studiengangsleiter: Christoph Markowsky

\section{SCHWEIZ (primärqualifizierend)}

40 Berner Fachhochschule - Fachbereich Gesundheit www.gesundheit.bfh.ch

Studienstandort: Basel oder Bern

Studiengangsleiter: Prof. Eugen Mischler (Bern), Dr. Heike Scheidhauer (Basel)

41 ZHAW Zürcher Hochschule für Angewandte Wissenschaften

www.gesundheit.zhaw.ch

Studiengangsleiterin: Cécile Ledergerber

42 Thim van der Laan University College Physiotherapy Landquart www.physioschule.ch, Studiengangsleiter: Ron Clijsen

\section{ÖSTERREICH (primärqualifizierend)}

43 IMC Fachhochschule Krems www.fh-krems.ac.at Studiengangsleiter: Reinhard Beikircher

44 FH Campus Wien www.fh-campuswien.ac.at Studiengangsleiterin: Silvia Mériaux-Kratochvila ME.d.

45 Fachhochschule St.Pölten www.fhstp.ac.at Studiengangsleiterin: FH-Prof. Mag. Astrid Figl-Hertlein, PT

46 Fachhochschule Salzburg www.fh-salzburg.ac.at Studiengangsleiterin: FH-Prof. Adelheid Gilmer

47 fhg - Zentrum für Gesundheitsberufe Tirol GmbH, Innsbruck www.fhg-tirol.ac.at Studiengangsleiter: Magister Claudia Wiederin

48 FH JOANNEUM, Graz www.fh-joanneum.at Studiengangsleiterin: Beate Salchinger, MMSc

49 Fachhochschule Kärnten, Klagenfurt www.fh-kaernten.at, Studiengangsleiterin: Andrea Umschaden 


\begin{tabular}{|c|c|c|c|c|c|c|c|}
\hline Abschluss & Studienform & Dauer und Beginn & $\begin{array}{l}\text { Kooperations- } \\
\text { schulen }\end{array}$ & seit/ab & Kosten & Plätze & $\begin{array}{l}\text { Credit } \\
\text { Points }\end{array}$ \\
\hline $\begin{array}{l}\text { Bachelor of } \\
\text { Physiotherapy (NL) }\end{array}$ & $\begin{array}{l}\text { ausbildungsintegriert } \\
\text { bzw. berufsbe- } \\
\text { gleitend für exam. PT }\end{array}$ & $\begin{array}{l}4 \text { Jahre bzw. } \\
3 \text { Jahre } \\
\text { im Oktober }\end{array}$ & ja & $\begin{array}{l}\text { Oktober } \\
2004\end{array}$ & insgesamt 5.500,- $€$ & 30 & 240 \\
\hline $\begin{array}{l}\text { Bachelor of } \\
\text { Physiotherapy (NL) }\end{array}$ & $\begin{array}{l}\text { ausbildungsintegriert } \\
\text { bzw. berufsbe- } \\
\text { gleitend für exam. PT }\end{array}$ & $\begin{array}{l}4,5 \text { Jahre bzw. } \\
26 \text { Monate } \\
\text { i.d.R. im Januar }\end{array}$ & ja & $\begin{array}{l}\text { Januar } \\
2009\end{array}$ & insgesamt $4.700-5.500,-€$ & 30 & 240 \\
\hline $\begin{array}{l}\text { Bachelor of } \\
\text { Physiotherapy (NL) }\end{array}$ & $\begin{array}{l}\text { ausbildungsintegriert } \\
\text { bzw. berufsbe- } \\
\text { gleitend für exam. PT }\end{array}$ & $\begin{array}{l}4 \text { Jahre bzw. } \\
26 \text { Monate } \\
\text { im Januar, Mai und } \\
\text { November }\end{array}$ & ja & 2007 & insgesamt 3.400-5.500,-€ & $25-30$ & 240 \\
\hline $\begin{array}{l}\text { Bachelor of } \\
\text { Physiotherapy (NL) }\end{array}$ & $\begin{array}{l}\text { ausbildungsintegriert } \\
\text { bzw. berufsbe- } \\
\text { gleitend für exam. PT }\end{array}$ & $\begin{array}{l}4 \text { Jahre bzw. } \\
29 \text { Monate } \\
\text { SS }\end{array}$ & ja & April 2007 & insgesamt $4.500-5.500,-€$ & 30 & 240 \\
\hline $\begin{array}{l}\text { Bachelor of } \\
\text { Physiotherapy (NL) }\end{array}$ & $\begin{array}{l}\text { ausbildungsintegriert } \\
\text { bzw. berufsbe- } \\
\text { gleitend für exam. PT }\end{array}$ & $\begin{array}{l}4 \text { Jahre bzw. } \\
30 \text { Monate } \\
\text { im März }\end{array}$ & ja & $\begin{array}{l}\text { März } \\
2008\end{array}$ & insgesamt $4.500-5.500,-€$ & $50-60$ & 240 \\
\hline
\end{tabular}

\begin{tabular}{|c|c|c|c|c|c|c|c|}
\hline Bachelor of Science & primärqualifizierend & $\begin{array}{l}3 \text { Jahre und } 10 \\
\text { Monate } \\
\text { in der KW } 38\end{array}$ & nein & $\begin{array}{l}\text { Sep- } \\
\text { tember } \\
2006\end{array}$ & 600,- CHF pro Semester & insgesamt 100 & 180 \\
\hline Bachelor of Science & $\begin{array}{l}\text { primärqualifizierend } \\
\text { (NC-Studiengang) }\end{array}$ & $\begin{array}{l}3 \text { Jahre und } 10 \\
\text { Monate } \\
\text { in der KW } 38\end{array}$ & nein & $\begin{array}{l}\text { Sep- } \\
\text { tember } \\
2006\end{array}$ & $\begin{array}{l}680,- \text { CHF pro Semester } \\
\text { plus } 500,- \text { CHF im Semester für } \\
\text { ausländische Studierende }\end{array}$ & 120 & 180 \\
\hline $\begin{array}{l}\text { Bachelor of } \\
\text { Physiotherapy (NL) }\end{array}$ & primärqualifizierend & $\begin{array}{l}4 \text { Jahre } \\
\text { Ende August }\end{array}$ & nein & 1990 & insgesamt 52.000,- CHF & 50 & 240 \\
\hline
\end{tabular}

Bachelor of Science primärqualifizierend

6 Semester
WS

nein WS 06/07 363,36€ pro Semester

40

180

Bachelor of Science primärqualifizierend

6 Semester

nein

WS $07 / 08 \quad 363,36 €$ pro Semester

115

WS

Bachelor of Science primärqualifizierend

6 Semester

nein

WS 06/07 $363,36 €$ pro Semester

Bachelor of Science primärqualifizierend

6 Semester

nein

WS 06/07 $363,36 €$ pro Semester

28

180 WS

Bachelor of Science primärqualifizierend

6 Semester

nein

WS $07 / 08 \quad 363,36 €$ pro Semester

26

180

WS

Bachelor of Science primärqualifizierend

6 Semester

nein

WS 06/07 keine Studiengebühren

70 